\title{
Neurosurgical management in lateral meningocele syndrome: case report
}

\author{
Erik C. Brown, MD, PhD, Kunal Gupta, MBBChir Cantab, PhD, and Christina Sayama, MD, MPH \\ Department of Neurological Surgery and Doernbecher Children's Hospital, Oregon Health \& Science University, Portland, \\ Oregon
}

\begin{abstract}
Lateral meningocele syndrome (LMS) is a rare genetic connective tissue disorder. It is associated with morphological changes similar to those of other connective tissue disorders, with the unique distinction of multiple, often bilateral and large, lateral meningoceles herniating through the spinal foramina. In some cases, these lateral meningoceles can cause pain and discomfort due to their presence within retroperitoneal tissues or cause direct compression of the spinal nerve root exiting the foramen; in some cases compression may also involve motor weakness. The presence of lateral meningoceles imposes unique challenges related to CSF flow dynamics, especially with concurrent Chiari malformation, which also occurs with increased frequency in individuals with LMS.

The authors present the case of a 6-month-old female with LMS with multiple lateral meningoceles throughout the thoracic and lumbar spine. The infant experienced a focal neurological abnormality due to enlargement of her lateral meningoceles following decompression of a symptomatic Chiari malformation and endoscopic third ventriculostomy. The finding was reversed through implantation of a ventriculoperitoneal shunt, which reduced the burden of CSF upon the lateral meningoceles. Such a case compels consideration that CSF flow dynamics in addition to altered connective tissue play a role in the presence of lateral meningoceles in patients within this and similar patient populations.
\end{abstract}

https://thejns.org/doi/abs/10.3171/2016.9.PEDS16311

KEY WORDS lateral meningocele syndrome; Lehman syndrome; lateral meningocele; shunt; Chiari malformation; endoscopic third ventriculostomy; congenital

$\mathrm{S}$ MALL meningeal diverticula of the spine may not be rare; limited observations report their occurrence in more than $10 \%$ of adults. ${ }^{16,18}$ When the diverticula are large, however, patients may present with neurological disturbance and subsequently require neurosurgical intervention. Such diverticula typically remain within the spinal canal, but they can, on occasion, herniate outside the spinal canal through the foramina. Symptoms that bring such cases to the attention of a neurosurgical specialist are often related to compression of nerve roots or the spinal cord itself. These symptoms can sometimes be positional, associated with increased pressure within and expansion of diverticula when the patient is upright relative to lying flat.

Lateral meningoceles of the spine are known to be associated with neurofibromatosis Type 1 (NF1) and connective tissue disorders, including Marfan's syndrome, Hajdu-Cheney syndrome, and Nevo syndrome, which have been correlated with Ehlers-Danlos syndrome Type VIA. ${ }^{8}$ Rarely, lateral meningoceles can occur primarily, as has been described in Lehman syndrome, also known as lateral meningocele syndrome (LMS). This syndrome was first described in $1977^{19}$ and more recently discovered to be due to dominant, often sporadic, truncating mutations of exon 33 of $\mathrm{NOTCH}^{7,10}$ (OMIM 130720). To our knowledge, a total of 14 cases identified as LMS have been previously described in the literature. ${ }^{1-5,7,9-11,14,19,22}$ It is possible that several other cases were described at a time when much less was known about LMS and that these cases were misidentified in original reports. . $^{6,2,20,21}$

We report on a 6-month-old female with severe and early-presenting LMS, requiring unique neurosurgical intervention. We discuss neurosurgical indications, management strategies, and clinical and imaging follow-up. We hope that our experience may be useful to future neurosurgeons who encounter patients with LMS.

ABBREVIATIONS CM-I = Chiari malformation Type I; CPAP = continuous positive airway pressure; $\mathrm{ETV}=$ endoscopic third ventriculostomy; LMS = lateral meningocele syndrome; NF1 = neurofibromatosis Type 1; VP = ventriculoperitoneal.

SUBMITTED May 27, 2016. ACCEPTED September 13, 2016.

INCLUDE WHEN CITING Published online December 2, 2016; DOI: 10.3171/2016.9.PEDS16311. 


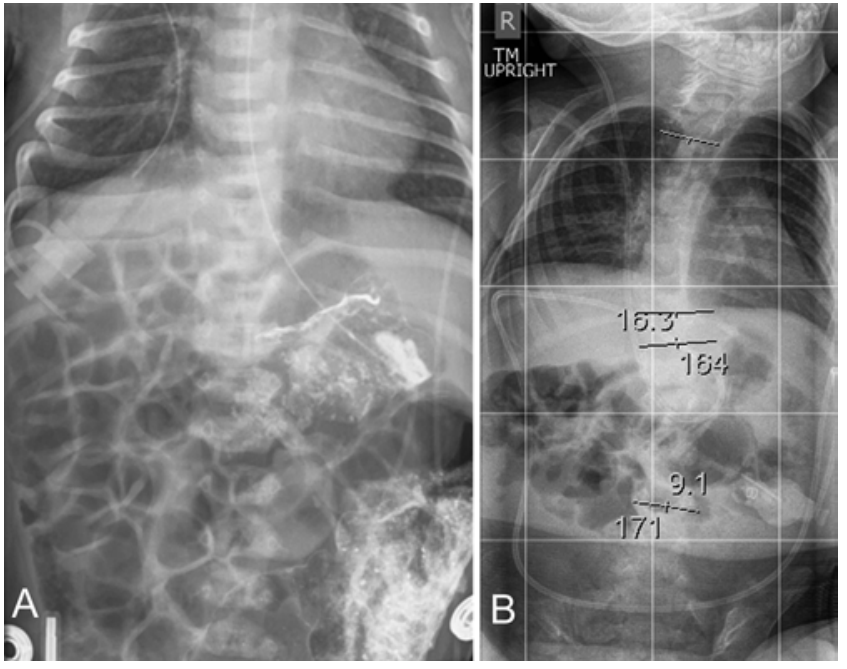

FIG. 1. Radiographs of the thoracolumbar region obtained when the patient was 6 weeks of age (A) and the total spine obtained when she was 17 months of age (B). At 6 weeks of age, the thoracolumbar spine was noted to be within normal limits. The radiograph obtained at 17 months of age demonstrates thoracic dextroscoliosis of $16^{\circ}$ with a compensatory lumbar levoscoliosis of $9^{\circ}$.

\section{Case Report}

History and Presentation

A 6-month-old female infant was referred to our neurosurgical team for consultation regarding an MRI finding of Chiari I malformation Type I (CM-I) with low-lying cerebellar tonsils. The MRI study had been performed while the patient hospitalized for complications related to refractory dysphagia with thin liquids and symptoms of choking and gagging at the age of 5 months. She had an ongoing history of failure to thrive and previous history of aspiration pneumonia requiring gastrostomy tube placement for supplemental feeding.

The patient's history included birth at 37 and 6/7 weeks via an uneventful normal spontaneous vaginal delivery to a genetically unremarkable 29-year-old mother and 34-yearold father (who also had 2 healthy children with a previous partner). At birth, she had a short umbilical cord, weighed $5 \mathrm{lb} 13 \mathrm{oz}$, was measured at 19.5 inches in length, and passed a newborn hearing screening test. Prenatally, she was noted to have increased nuchal thickness, hemivertebra at L-2 on prenatal ultrasonography, and normal findings of a prenatal chromosomal microarray analysis based on chorionic villous sampling; a postnatal chromosomal microarray analysis was also negative for abnormality. She had developed malrotation of the intestine, and this was treated with a Ladd procedure when she was 4 months of age. A spinal radiograph obtained when she was 6 weeks old suggested a normal lumbar spine (L1-5, Fig. 1A).

The family history was notable for 4 first-trimester miscarriages and a healthy 2-year-old boy, who had been born to the couple prior to the birth of this girl. The father reported that he himself was a "slow learner" and had required speech therapy in childhood.

Brain and spine MRI at 5 months of age revealed CM-I, with extremely low-lying cerebellar tonsils pegged to the level of C-4 (Fig. 2A), and multiple lateral meningoceles (Fig. 3) of the thoracic and lumbar spine and posteriorly scalloped vertebral bodies. The cervical spinal cord was anteriorly displaced, although there was no cord signal change and a syrinx was not present. There was no overt hydrocephalus, but ventricles were noted to be larger than average. The patient was referred to pediatric neurosurgery for consultation. The largest lateral meningocele protruded from the left intervertebral foramen at the L1-2 interspace, initially measuring $1.7 \mathrm{~cm} \times 2.6 \mathrm{~cm}$ in the axial plane; the conus was noted to be at the L2-3 interspace.

Her CM-I was considered to be symptomatic, causing refractory dysphagia, choking, and aspiration, and thus surgical decompression was indicated. Her head circumference (Fig. 4) was noted to have jumped percentiles, despite a flat fontanelle, well-approximated sutures, and lack of other clinical signs of hydrocephalus such as sunsetting eyes in downward gaze, lethargy, or vomiting; her head circumference was at the 12 th percentile at 1 month
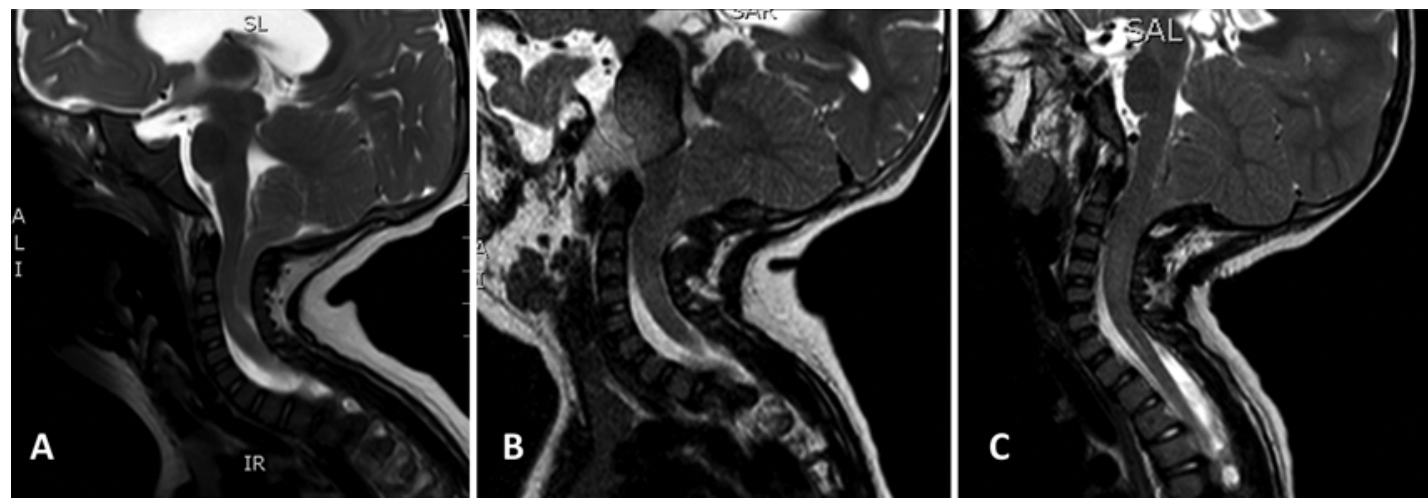

FIG. 2. T2-weighted MR images obtained in the midline sagittal plane when the patient was 5 (A), 9 (B), and 16 (C) months of age. At age 5 months $(A)$, the cerebellar tonsils can be observed to extend down to the level of $C-4$ with compression of the cervical spinal cord; it is notable that there is no visible central canal at this time. At age 9 months (B), over 2 months after Chiari decompression, the cerebellar tonsils can be observed to extend only as far as $\mathrm{C}-2$, an improvement from preoperative imaging. At age 16 months $(\mathrm{C})$, over 5 months following right VP shunt placement there is stable herniation of the cerebellar tonsils to $\mathrm{C}-2$. This examination is notable for a new finding of a narrow central canal starting at C-7, as seen here. This canal continues down to T-11, not shown here, and has a maximum diameter of $2 \mathrm{~mm}$. 

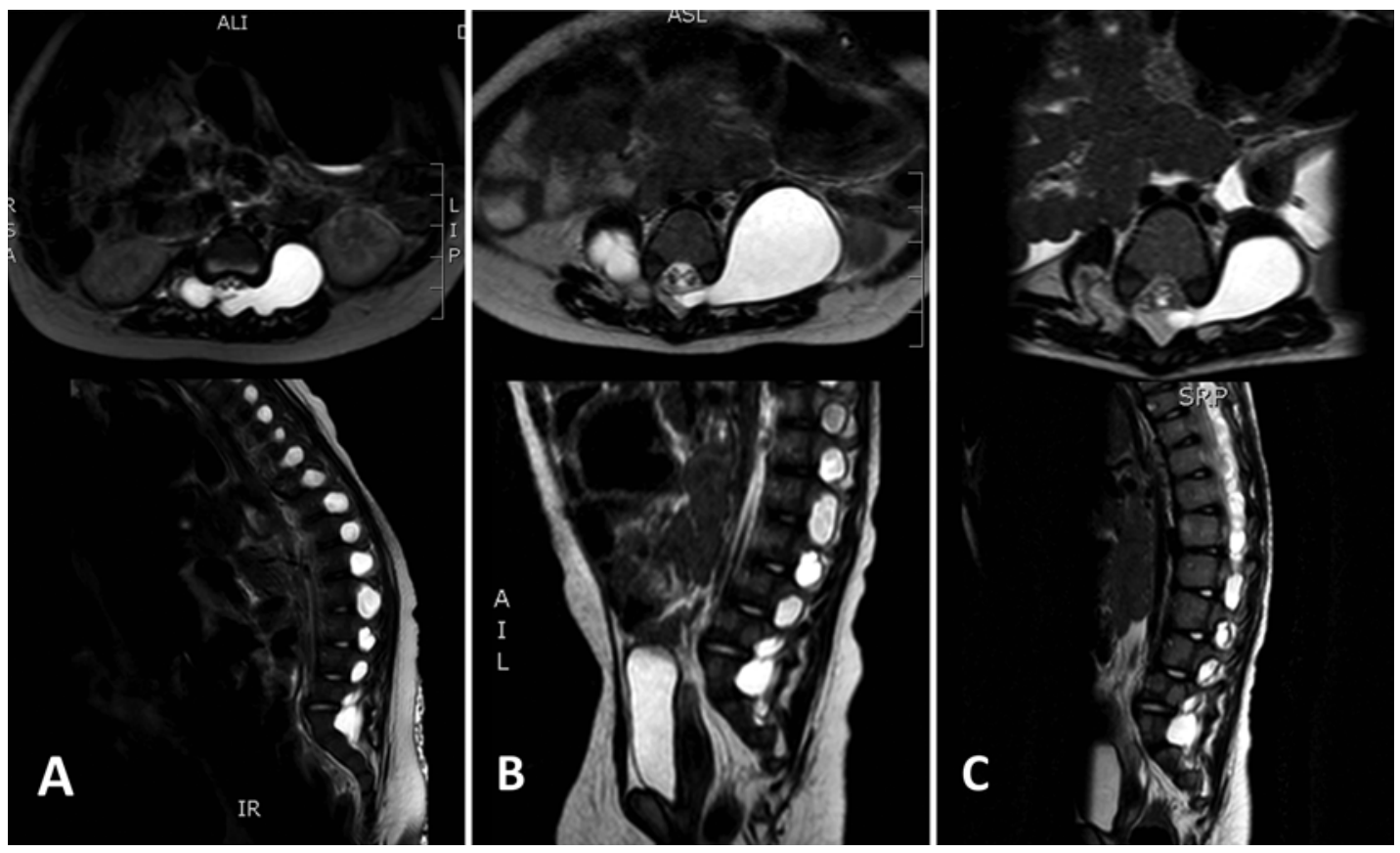

FIG. 3. T2-weighted MR images of the spine in the axial (upper row) plane at the level of the L1-2 interspace and left-biased parasagittal (lower row) plane at 5 (A), 9 (B), and 16 (C) months of age. At age 5 months (A), it can be observed that there are multiple lateral meningoceles herniating through enlarged foramina. The largest of these herniating through the enlarged L-1 foramina can be seen in the axial plane, measuring here to be $1.7 \times 2.6 \mathrm{~cm}$. There is a clear, widely patent communication between the lateral meningocele and the thecal sac, demonstrating that this meningocele is indeed filled with freely flowing CSF, the conus was noted to be at the level of L2-3. At age 9 months (B), over 2 months after Chiari decompression, the left-side lateral meningocele at the L-1 foramen is shown to be enlarged compared with the previous image; now measuring $2.9 \times 4.1 \mathrm{~cm}$, the conus was noted to be stable at the level of L2-3 (not shown). At 16 months (C), over 5 months after right VP shunt placement, the previously enlarged lateral meningocele at the left L-1 foramina can be seen to have regressed to a size of $1.8 \times 2.7 \mathrm{~cm}$, more similar to the pre-decompression size despite the patient's corporeal growth with advancing age, the conus was noted to be stable at the level of $L 2-3$ (not shown).

of age, 76th percentile at 4 months of age, and 89th percentile at 6 months of age. Intradural decompression of the CM-I was delayed until 7.5 months of age due to multiple infectious processes, including upper respiratory infection and gastroenteritis; the ventricles were observed to undergo a small amount of enlargement during this time (Fig. 5), while the patient's neurological examination remained unchanged and her head circumference stayed at the 94th percentile (Fig. 4).

\section{First Operation and Postoperative Course}

The patient underwent an endoscopic third ventriculostomy (ETV), suboccipital craniectomy, C1-3 laminectomies, tonsillar coagulation, and expansile duraplasty without complication at 7.5 months of age; her dura mater was noted to be roughly 3-4 times thicker than normal. An ETV was included in the operation in an attempt to treat her worsening ventriculomegaly and head growth in addition to her CM-I, as it was unclear which condition was driving the other; at our institution, ETV is generally attempted when possible in an effort to avoid shunt placement and the associated long-term implications. Her case had also been discussed prior to any surgical intervention in our pediatric neurosurgery conference.

At 2 weeks' follow-up, her parents reported that her oral intake had improved, her choking and gagging were

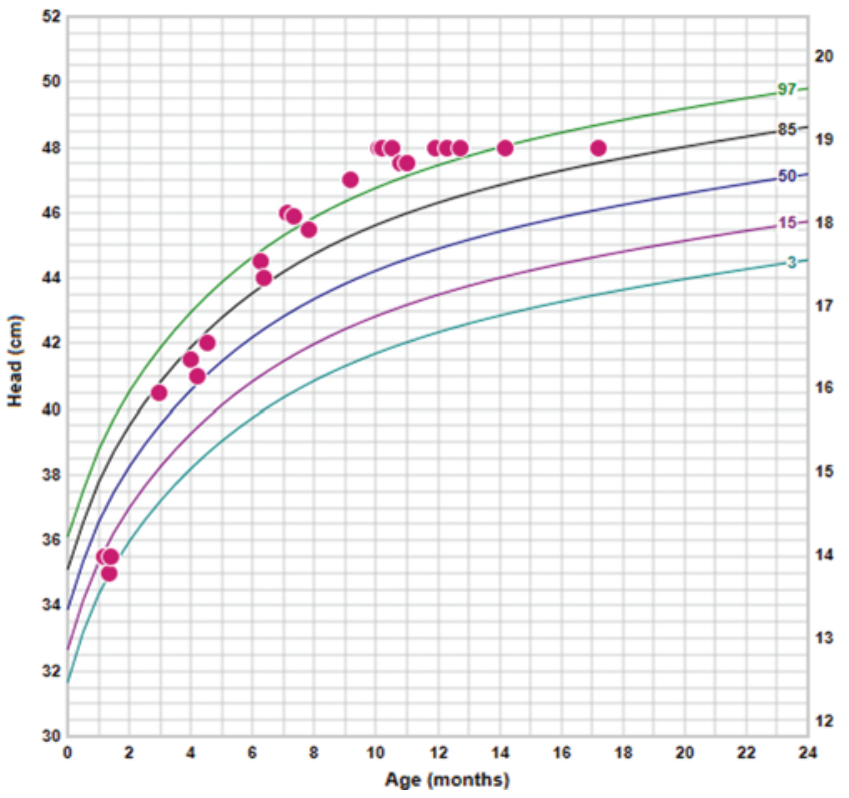

FIG. 4. The patient's head circumference growth chart, based on that promoted by the World Health Organization. Despite stable ventricle size after Chiari decompression and ETV at age 7.5 months, the patient's head circumference continued to increase until VP shunt placement at age 9 months. Since that time, her head circumference has shown a normalizing trend. Figure is available in color online only. 

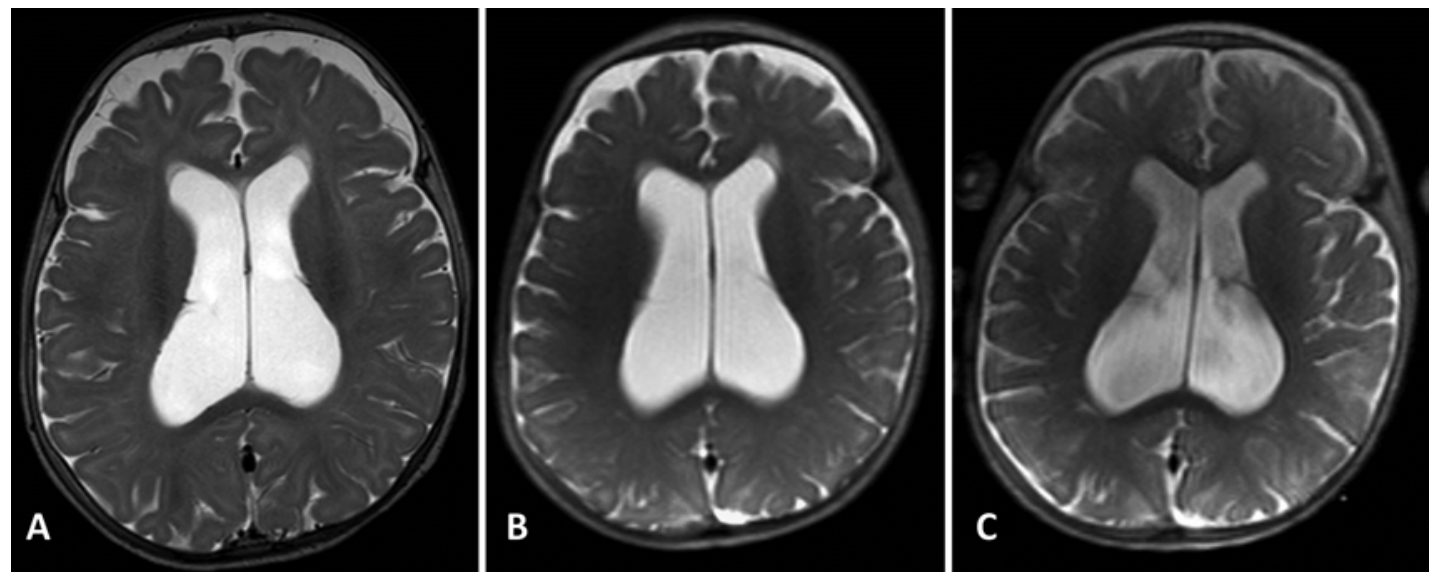

FIG. 5. Axial T2-weighted MR images obtained when the patient was $5(\mathbf{A}), 6(\mathbf{B})$, and $9(\mathbf{C})$ months of age. At age 5 months (A), there is some dilation of the ventricles with mild T2 changes along the bifrontal periventricular region thought to possibly represent transependymal flow versus myelination differences in an infant. At age 6 months (B), the ventricles are slightly increased from the prior size with slight increase in T2 changes along the bifrontal periventricular region, now thought to be more consistent with transependymal flow. At age 9 months (C), over 2 months after Chiari decompression, the ventricles are slightly decreased, and there is less T2 signal along the bifrontal periventricular region, suggesting improvement of hydrocephalus.

reduced, and she had begun to tolerate solid food. However, about 5-6 weeks after her CM-I decompression and ETV, the parents noted that the patient was becoming irritable when upright and that she was showing a preference for use of the right leg. Upon examination, the parents' complaints were reproduced, as the patient was observed to be comfortable moving all extremities when lying flat and became extremely irritable with the left leg held in a flexed position when upright. Another spine MRI study was performed when the patient was 9 months old; this study revealed enlargement of the lateral meningoceles, especially the lesion protruding from the left L1-2 interspace, which now measured $2.9 \mathrm{~cm} \times 4.1 \mathrm{~cm}$ in the axial plane (Fig. 3B). The patient's lateral ventricles were stable in size while her head circumference had increased slightly and was now greater than the 99th percentile. After much deliberation with colleagues within and and outside our institution, it was decided to place a ventriculoperitoneal (VP) shunt to attempt to reduce the CSF burden upon the lateral meningoceles.

\section{Second Operation and Postoperative Course}

VP shunt placement with a nonprogrammable mediumpressure valve was performed at 10.5 months of age. The patient tolerated the procedure well. At 2 weeks' followup, the parents reported that the patient was doing well, sitting comfortably and playful with full strength and range of motion of all extremities when upright.

\section{Genetic Evaluation}

Subsequent genetic evaluation and testing revealed the presence of a heterozygous, de novo 2-nucleotide deletion (more specifically: c.6659_6660delAG) in exon 33 on the NOTCH3 gene, definitively identifying LMS; other specific genetic testing was also completed for $M I D 1, F B N 1$, $M Y L K, S M A D 3, T G F \beta 2, T G F \beta R 1$, and TGF $\beta R 2$, which were all found to be without mutation or with variants expected to be benign.

\section{Additional Treatment and Follow-Up}

At 12 months of age, the patient underwent surgical correction of cleft palate with Furlow palatoplasty and placement of bilateral tympanostomy tubes for treatment of middle ear effusions related to eustachian tube dysfunction. She began to experience severe apnea during sleep, which upon multidisciplinary workup was determined to be due to velopharyngeal and oropharyngeal collapse on inspiration, likely unrelated to her intact palatal repair, and was treated with supplemental oxygen during sleep. Her respiratory function was compromised further during an upper respiratory infection at 15 months of age, which resolved with antibiotics and supportive care. Following resolution of this episode, she continued to demonstrate nightly oxygen saturation levels as low as the $80 \%$ range due to her sleep apnea and was determined appropriate for continuous positive airway pressure (CPAP) treatment by our institution's pediatric sleep specialists following the finding of a worsening of her Apnea Hypopnea Index value from 30 to 50 . Her polysomnogram was consistent with severe obstructive sleep apnea, and there was no evidence to suggest any component of central sleep apnea. There was no evidence of shunt malfunction; her ventricle size remained stable, and her head growth had begun to decelerate. The otolaryngologist did not note any vocal cord weakness or paralysis. The pulmonology and otolaryngology specialists recommended tracheostomy if CPAP treatment fails. As there was no central etiology identified for this new obstructive sleep apnea, further decompression was not recommended as treatment.

On physical examination at 17 months of age, the patient had a small soft cleft palate (previously confirmed by nasopharyngeal reflux on barium swallow testing), dolichocephaly, bitemporal narrowing, a flattened facial profile, hypertelorism with telecanthus, unilateral left eye ptosis, narrow external ear canals, posteriorly rotated lowset ears, micrognathia, a thin vermillion border, a smooth philtrum, facial and corporeal hypotonia, hyperextensible 
joints, rounded feet, and vesicoureteral reflux of Grade 2 on the right and Grade 3 on the left (Fig. 6).

At 7 months after shunt placement, the patient remained free of her preoperative neurological symptoms; it was notable that no keloid or hypertrophic scar (common in patients with LMS) had formed at any of her surgical incisions. She remained without signs or symptoms of hydrocephalus, such as headaches, lethargy, vomiting, or sun-setting eye sign. Indeed, she was playful and interactive. Her head circumference had begun to normalize and was measured to be at the 90th percentile for age. Of note, her body length had always tracked along the third percentile growth curve while her weight, below the third percentile prior to 4 months of age, but at this evaluation at 17 months of age it was tracking along the 15 th percentile growth curve. She was noted to display some thoracic kyphosis on physical examination (Fig. 6C), although this finding may be due to truncal hypotonia, and we obtained baseline total spine radiographs due to her risk of development of scoliosis, described below. The patient did not appear at this time to have any symptoms suggestive of tethered cord, and therefore the decision was made in favor of careful observation due to her multiple medical problems, respiratory issues, complex CSF dynamics, and connective tissue disorder. The patient was continuing to show gradual improvement in her abilities for oral intake using her gastrostomy tube only for nutritional supplementation as needed. MRI of her spine performed at 16 months of age showed improvement in her lateral meningoceles from previous imaging (Fig. 5C), with the largest protruding from the left L1-2 interspace measuring 1.8 $\mathrm{cm} \times 2.7 \mathrm{~cm}$, which was smaller compared with imaging prior to shunt placement. The conus was stable at the inferior endplate of L-2, and there was the new observation of a prominent cervical and thoracic central canal, potentially representing a developing syrinx, measuring $2 \mathrm{~mm}$ in maximal diameter. She also had developed a thoracic dextroscoliosis of $16^{\circ}$ with a compensatory lumbar levoscoliosis of $9^{\circ}$ (Fig. 1B). It is notable that the cerebellar tonsils were extending to the bottom of C-2, and although she was clinically and radiographically improved from her preoperative CM-I decompression, there was still radiological evidence of "crowding" at the craniocervical junction.

Our institution's physical therapy team has consistently followed the patient over time working with the patient and family regarding her mild developmental delay, primarily a gross motor delay with progress using exercises. At her last visit with physical therapy at 18 months of age she was crawling on her own more, able to walk with handheld assistance, and she climbed her first set of stairs.

\section{Discussion}

We report here a case of LMS requiring intensive neurosurgical observation and multiple neurosurgical interventions. The most distinctive features of LMS include lateral meningoceles herniating through the intervertebral foramina and associated scalloping of the posterior vertebral bodies. This features more prominently in a dependent manner, in the lower thoracic and lumbar spine, unlike in NF1 where the lateral meningoceles, when they occur, are more commonly observed in the thoracic spine. ${ }^{24}$ Common features shared with other connective tissue disorders ${ }^{3,9,11}$ include dolichocephaly with high forehead, shallow supraorbital ridges, high arched eyebrows, ptosis, hypertelorism, elongated and flattened philtrum, tented and thin upper lip, high and narrow or cleft palate, micro- or retrognathia, wormian bones, thickened skull, scoliosis, and low-set posteriorly angulated ears. The posterior hairline is low, with a coarse texture to the hair itself. Hyperextensibility with ligamentous laxity and muscular hypotonia, often including the facial muscles, are also quite common in patients with this syndrome and are thought to be the root cause of any motor developmental delay that occurs, ${ }^{10}$ as cognition is not primarily affected in the majority of cases. Relevant to this case report, $\mathrm{CM}-\mathrm{I}^{4,7}$ has been previously reported in 2 patients and tethered cord ${ }^{10}$ in another patient.
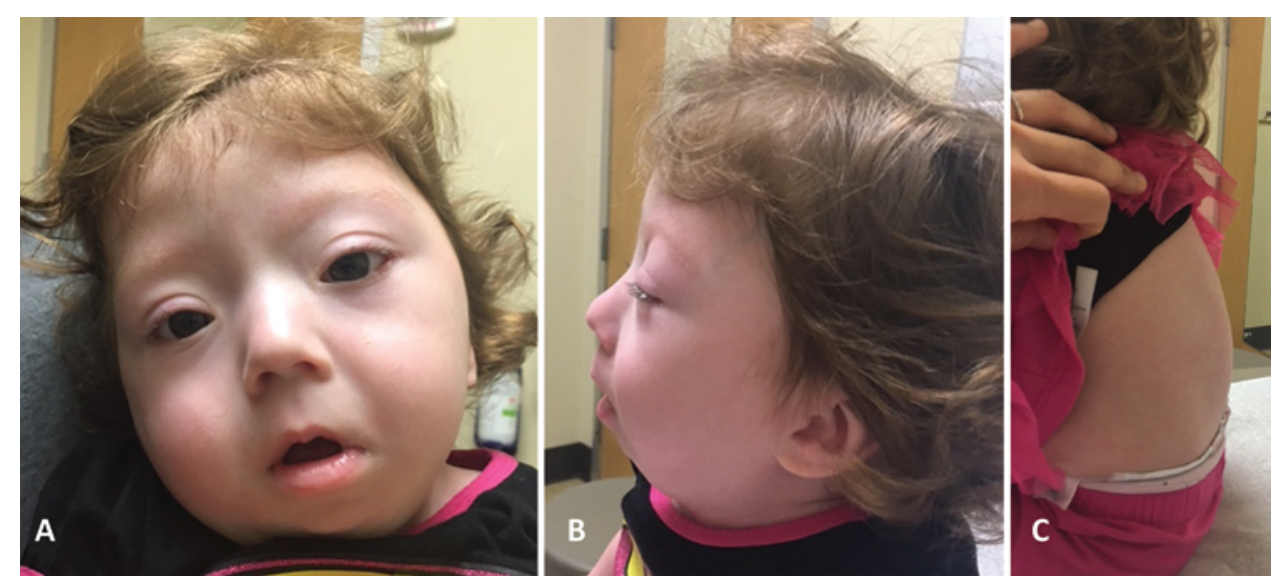

FIG. 6. Photographs of the patient at age 17 months following the procedures described herein. The anterior view (A) demonstrates mild left eye ptosis, a medial canthal distance measuring $3.5 \mathrm{~cm}$ (indicating telecanthus ${ }^{17}$ ), a smooth philtrum, and a thin upper vermillion border. The lateral view of the child's face (B) shows micrognathia, dolichocephaly with high forehead, flattened facial profile, and posteriorly rotated low-set ears. The lateral view of the patient's back while seated (C) demonstrates mild hyperkyphosis, which may represent an early sign of the development of scoliosis. Published with parental consent. Figure is available in color online only. 
Lateral meningoceles are often associated with connective tissue disorders and NF1 and also manifest as a primary disorder in LMS. These are often followed clinically with radiographic observation, ${ }^{6,10,13}$ surgical intervention being reserved for those that become symptomatic. Indications for intervention in lateral meningoceles are poorly defined. Clinical symptoms that guided our indications for surgery included pain likely related to radicular symptoms or nerve compression from larger meningoceles and a pressure-related phenomenon when upright, with concomitant radiographic findings of grossly enlarged lateral meningoceles at multiple levels. Weakness can also occur and is an indication for surgical intervention, but other localizing lesions must be excluded, as other central nervous system abnormalities may confound the clinical milieu. In the pediatric population, pain can manifest as poor utilization of a limb and pathological handedness. Furthermore, weakness and pain can manifest as gross motor milestone regression and may prompt surgical intervention. The patient described herein had no other localizing lesion to explain the decreased use of her left leg, which also improved with CSF shunting, suggesting that the enlarged meningoceles were contributing to her leg use preferences. Rare case reports of cardiac compression due to large thoracic meningoceles have also been reported. ${ }^{15}$ Axial back pain has occasionally resulted in surgical intervention for lateral meningoceles, often with poor outcomes, and paindirected therapies such as TENS (transcutaneous electrical nerve stimulation) or spinal cord stimulation may be associated with better outcomes in such cases. ${ }^{3,24}$

Although in several of the cases of LMS reported in the literature the patients have undergone neurosurgical decompression for relief of symptoms, neurosurgical options and management of these patients has been poorly defined. In a 1978 report by Katz et al. and in subsequent reports by Chen et al. and Alves et al., it was discussed that patients with lateral meningoceles should undergo treatment if symptomatic; however neither management methods nor symptomatology indicating intervention were discussed. ${ }^{1,414}$ Castori et al. reported on a 55-year-old female patient with back pain and radiculopathy associated with lateral meningoceles; this patient underwent resection of the lesions and unfortunately had a poor outcome, with bladder and bowel dysfunction as well as worsened weakness; the operative procedure was not described. ${ }^{3}$ Rainov et al. described laminectomy and ligation as the primary means for surgically treating lateral meningoceles in NF1, but reported a lack of options in the presence of multiple, broad-based meningoceles-large isolated and symptomatic meningoceles have rarely been treated with laminectomy and resection of the meningocele..$^{24}$ Risks associated with this treatment include destabilization of the spine and the requirement for instrumented spinal fusions, and patients with LMS may be at increased risk due to the presence of bony changes of the spine such as posteriorly scalloped vertebrae and scoliosis. Cerebrospinal fluid leak is also of great concern with primary repair or when patched repair is required. Furthermore, these procedures are inadvisable in the presence of multiple meningoceles as well as meningoceles with large broad-based dural pedicles, which are often present in LMS. Gripp et al. reported on a patient with LMS and CM-I associated with hydrocephalus with ventriculomegaly; ${ }^{11}$ VP shunt placement, Chiari decompression, and shunting of a thoracic meningocele to the pleura were performed. Again, surgical indications, as well as radiographic and clinical sequelae, were not fully discussed. A second case of CM-I in LMS described in the literature ${ }^{7}$ did not elaborate upon any neurosurgical intervention or follow-up.

We observed our patient closely and noted worsening radiological enlargement of her meningoceles, correlating with objective gross motor regression. We also noted that her symptoms were positional, being worse when she was upright. Given the dependent location of her meningoceles, we determined that her neurological symptoms were likely attributable to her enlarging meningoceles. Evaluation of the meningoceles revealed them to be multiple and broad based, largely precluding resection, ${ }^{24}$ which would entail a high risk of CSF leak and surgical destabilization as well as meningocele recurrence. Single cases treated with CSF diversion by VP and lumboperitoneal shunting have been reported individually, but with little to no outcome data. ${ }^{11,22}$ Here, we report the use of CSF diversion for symptomatic lateral meningoceles in a 10-month-old patient, with placement of a VP shunt; this is unlike the shunt described by Gripp et al., ${ }^{11}$ which was placed to treat hydrocephalus prior to Chiari decompression. At 2 weeks' follow-up, our patient's parents reported improved movement, as well as reduced irritability when she was upright. This continued to be true at 7 months' follow-up, when her parents reported continued symmetrical motor function in all extremities. Our institution's physical therapy team also noted continued improvement in the use of both lower extremities, and the patient is now starting to ambulate. On MRI we noted improvement of her lateral meningoceles, which had returned to a size similar to that prior to Chiari decompression.

In the presence of broad-based and multiple symptomatic, enlarging lateral meningoceles, we recommend consideration of CSF diversion by placement of a VP shunt. It may be speculated that CSF shunting will not be beneficial if the underlying problem is a connective tissue disorder, but this case clearly shows that lateral meningoceles can improve in a child with just such an abnormality. Shunt placement may also assist with and improve the safety of CM-I decompression in LMS by reducing supratentorial intracranial pressure following decompression so as to relieve otherwise increased stress upon the lateral meningoceles of the lumbar spine. Endoscopic ventriculostomy in this case was not an effective management strategy to treat the patient's abnormal CSF flow dynamics and could possibly have contributed to the worsening of her lateral meningoceles. Indeed abnormal CSF flow dynamics in CM-I have been demonstrated to be associated with syrinx development, ${ }^{23}$ increased systolic CSF flow duration in the ventral spinal subarachnoid space particularly. The effect of the lateral meningoceles themselves upon CSF dynamics is not well studied, although they can reasonably be expected to further contribute to as well as be influenced by departures from normal flow patterns. The long-term effects of ventricular CSF diversion upon the natural history of lateral meningoceles of the thoracic and 
lumbar spine are unknown and require further investigation. Patients such as the child in the present case will need ongoing long-term follow-up, as they may have multiple neurosurgical conditions. It will be important to determine whether shunting slows or eliminates long-term progression of lateral meningoceles and provides long-term relief of symptoms without need for further surgical intervention. Our patient will also require ongoing follow-up of her neuromuscular scoliosis, which will likely progress, given her underlying hypotonia. Furthermore, we recommend screening for $\mathrm{CM}-\mathrm{I},{ }^{7}$ tethered cord, syrinx, scoliosis, and close observation for hydrocephalus in patients with known or suspected LMS, in addition to evaluation for symptomatic as well as radiological progression of lateral meningoceles during preoperative planning and postoperative care.

\section{Acknowledgments}

We would like to acknowledge the parents of the child reported herein for their approval of our submission of this case report and their continued commitment to finding the very best care for their daughter. We thank Shirley McCartney, PhD, for her helpfulness in final edits to this manuscript.

\section{References}

1. Alves D, Sampaio M, Figueiredo R, Leão M: Lateral meningocele syndrome: additional report and further evidence supporting a connective tissue basis. Am J Med Genet A 161A:1768-1772, 2013

2. Avela K, Valanne L, Helenius I, Mäkitie O: Hajdu-Cheney syndrome with severe dural ectasia. Am J Med Genet A 155A:595-598, 2011

3. Castori M, Morlino S, Ritelli M, Brancati F, De Bernardo C, Colombi M, et al: Late diagnosis of lateral meningocele syndrome in a 55-year-old woman with symptoms of joint instability and chronic musculoskeletal pain. Am J Med Genet A 164A:528-534, 2014

4. Chen KM, Bird L, Barnes P, Barth R, Hudgins L: Lateral meningocele syndrome: vertical transmission and expansion of the phenotype. Am J Med Genet A 133A:115-121, 2005

5. Correia-Sá I, Horta R, Neto T, Amarante J, Marques M: Lehman syndrome: a new syndrome for Pierre Robin sequence. Cleft Palate Craniofac J 52:369-372, 2015

6. de Oliveira RS, Amato MC, Santos MV, Simão GN, Machado HR: Extradural arachnoid cysts in children. Childs Nerv Syst 23:1233-1238, 2007

7. Ejaz R, Qin W, Huang L, Blaser S, Tetreault M, Hartley T, et al: Lateral meningocele (Lehman) syndrome: a child with a novel NOTCH3 mutation. Am J Med Genet A 170A:10701075,2016

8. Giunta C, Randolph A, Al-Gazali LI, Brunner HG, Kraenzlin ME, Steinmann B: Nevo syndrome is allelic to the kyphoscoliotic type of the Ehlers-Danlos syndrome (EDS VIA). Am J Med Genet A 133A:158-164, 2005

9. Gripp KW: Lateral meningocele syndrome and HajduCheney syndrome: different disorders with overlapping phenotypes. Am J Med Genet A 155A:1773-1775, 2011

10. Gripp KW, Robbins KM, Sobreira NL, Witmer PD, Bird LM, Avela K, et al: Truncating mutations in the last exon of NOTCH3 cause lateral meningocele syndrome. Am J Med Genet A 167A:271-281, 2015

11. Gripp KW, Scott CI Jr, Hughes HE, Wallerstein R, Nicholson L, States L, et al: Lateral meningocele syndrome: three new patients and review of the literature. Am J Med Genet 70:229-239, 1997

12. Isono M, Hori S, Konishi Y, Kinjo H, Kakisako K, Hirose R, et al: Ehlers-Danlos syndrome associated with multiple spinal meningeal cysts - case report. Neurol Med Chir (Tokyo) 39:380-383, 1999

13. Jeong JW, Park KY, Yoon SM, Choe W, Kim CH, Lee JC: A large intrathoracic meningocele in a patient with neurofibromatosis-1. Korean J Intern Med 25:221-223, 2010

14. Katz SG, Grünebaum M, Strand RD: Thoracic and lumbar dural ectasia in a two-year-old boy. Pediatr Radiol 6:238240, 1978

15. Kim YJ, Cho HM, Yoon CS, Lee CK, Lee TY, Seok JP: Surgical treatment of thoracic menigocele associated with neurofibromatosis and kyphoscoliosis. Korean J Thorac Cardiovasc Surg 44:383-386, 2011

16. Kranz PG, Stinnett SS, Huang KT, Gray L: Spinal meningeal diverticula in spontaneous intracranial hypotension: analysis of prevalence and myelographic appearance. AJNR Am J Neuroradiol 34:1284-1289, 2013

17. Laestadius ND, Aase JM, Smith DW: Normal inner canthal and outer orbital dimensions. J Pediatr 74:465-468, 1969

18. Larsen JL, Smith D, Fossan G: Arachnoidal diverticula and cystlike dilatations of the nerve-root sheaths in lumbar myelography. Acta Radiol Diagn (Stockh) 21 (2A):141-145, 1980

19. Lehman RA, Stears JC, Wesenberg RL, Nusbaum ED: Familial osteosclerosis with abnormalities of the nervous system and meninges. J Pediatr 90:49-54, 1977

20. Marbacher S, Barth A, Arnold M, Seiler RW: Multiple spinal extradural meningeal cysts presenting as acute paraplegia. Case report and review of the literature. J Neurosurg Spine 6:465-472, 2007

21. Merle P, Georget AM, Goumy P, Jarlot D: Primary empty sella turcica in children. Report of two familial cases. Pediatr Radiol 8:209-212, 1979

22. Philip N, Andrac L, Moncla A, Sigaudy S, Zanon N, Lena G, et al: Multiple lateral meningoceles, distinctive facies and skeletal anomalies: a new case of Lehman syndrome. Clin Dysmorphol 4:347-351, 1995

23. Pinna G, Alessandrini F, Alfieri A, Rossi M, Bricolo A: Cerebrospinal fluid flow dynamics study in Chiari I malformation: implications for syrinx formation. Neurosurg Focus 8(3):E3, 2000

24. Rainov NG, Heidecke V, Burkert W: Thoracic and lumbar meningocele in neurofibromatosis type 1 . Report of two cases and review of the literature. Neurosurg Rev 18:127-134, 1995

\section{Disclosures}

The authors report no conflict of interest concerning the materials or methods used in this study or the findings specified in this paper.

\section{Author Contributions}

Conception and design: Sayama. Acquisition of data: all authors. Analysis and interpretation of data: all authors. Drafting the article: all authors. Critically revising the article: all authors. Reviewed submitted version of manuscript: Sayama, Brown. Approved the final version of the manuscript on behalf of all authors: Sayama.

\section{Correspondence}

Christina Sayama, Neurological Surgery, Oregon Health \& Science University, 3303 SW Bond Ave., Portland, OR 97239. email: sayama@ohsu.edu. 